Published by Al-Nahrain College of Medicine P-ISSN 1681-6579

E-ISSN 2224-4719

Email: iraqijms@colmed-alnahrain.edu.iq

http://www.colmed-alnahrain.edu.iq

http://www.iraqijms.net

Iraqi JMS 2018; Vol. 16(3)

\title{
Immunohistochemical Expressions of PAX8 in Ovarian Surface Epithelial Tumors
}

\author{
Samar A. Al-Shami ${ }^{1}$ MSC, Ban J. Qasim ${ }^{1}$ PhD, Ahmed M. Hassan ${ }^{2}$ MSC \\ ${ }^{1}$ Dept. of Pathology and Forensic Medicine, College of Medicine, Al-Nahrain University, Baghdad, Iraq. ${ }^{2}$ Maissan Health \\ Directorate, Maissan, Iraq
}

\begin{abstract}
Background PAX8 is a nuclear transcription factor with limited expression in normal and neoplastic tissues, it has the potential to induce tumorigenesis and is expressed in a tissue during neoplastic transformation. PAX8 is a useful marker to distinguish gynecologic cancers from non-gynecologic malignancies.

Objective

Methods

To assess the immunohistochemichal expression of PAX8 in ovarian surface epithelial tumors.

This study included a total of 100 ovarian tissue paraffin blocks, 70 tissue paraffin blocks of ovarian tumors obtained from patients who underwent total abdominal hysterectomy and bilateral salpingooophorectomy and 30 tissue paraffin blocks were the control group included normal ovarian tissue and fallopian tubes tissue. From each paraffin block, 2 sections were taken, one was stained with the routine hematoxylin and eosin stain and the other section was stained immunohistochemically for PAX8.

Results PAX8 showed a high significant difference in its immunohistochemical expression between the control group and the case group with the highest expression in malignant ovarian surface epithelial tumors $(\mathrm{P}<$ 0.001). Also, PAX8 showed a highly significance in the expression of PAX8 in relation to histopathological types $(P<0.001)$ except for the mucinous tumors, which showed statistically non-significance $(P=$ $0.641)$, also, a highly significant relation with the increment of tumor grade $(r=0.769, P<0.001)$. PAX8 showed a non-significant relation with increase of the age $(r=0.147, P=0.225)$, with tumor stage $(r=$ $0.433, P=0.057)$, with the presence of ascites $(P=0.446)$.

Conclusion The highly significant differences in the immunohistochemical expression of PAX8 in the ovarian surface epithelial tumors tissues compared to the control groups reflect its important role in ovarian tumorigenesis. Besides PAX8 can be used as an important marker for discriminating ovarian nonmucinous from mucinous tumors. Also, there is a possible role of PAX8 in the development and differentiation of ovarian malignant surface epithelial tumor.

Keywords Ovarian surface epithelial tumors, ovarian carcinoma, transcription factor, paired box antigen, PAX8, immunohistochemistry

Citation Al-Shami SA, Qasim BJ, Hassan AM. Immunohistochemical expressions of PAX8 in ovarian surface epithelial tumors. Iraqi JMS. 2018; 16(3): 298-307. doi: 10.22578/IJMS.16.3.9
\end{abstract}

List of abbreviations: $\mathrm{CIC}=$ Cortical inclusion cysts, $\mathrm{EMT}=$ Epithelial-mesenchymal transition, FIGO = Federation of gynecology and obstetrics, H\&E = Hematoxylin \& Eosin staining, HGSC = High grade serous carcinoma, LGSC $=$ Low grade serous carcinoma, OCCC $=$ Ovarian clear cell carcinoma, STIC = Serous tubal intra-epithelial carcinoma, TCC $=$ Transitional cell carcinoma, WT-1 = Wilms tumor 1 protein

\section{Introduction}

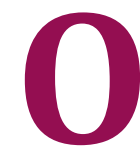
varian cancer represents the $6^{\text {th }}$ most commonly diagnosed cancer among women around the world, and each year it cause more deaths than any other cancer of the female reproductive system ${ }^{(1)}$. It is found to be the $2^{\text {nd }}$ most common gynecological malignancy in the United States, and it is the leading cause of death in more than 13,000 each year in the United States. The risk that a woman can develops ovarian cancer is $1 / 71^{(2,3)}$. In Iraq, ovarian tumors rank the $6^{\text {th }}$ commonest cancer among females and 
constituted $4.1 \%$ according to latest published Iraqi Cancer Board Registry in $2011^{(4)}$.

Surface epithelial tumors represent about 90\% of primary ovarian tumors, which can be found solid, cystic or mixture of both, they can be benign, borderline or malignant. These tumors represent $15 \%$ of all epithelial ovarian cancers, with the serous and mucinous types making up the vast majority of cases ${ }^{(5)}$. The ovarian surface epithelium develops from the coelomic epithelium (mesothelium) that covering the embryonic gonad. This epithetlium is continuous with the coelomic epithelium that penetrates the underlying mesenchyme to form the Müllerian duct. This embryonic close proximity is reflected in the various directions of Müllerian differentiation, which manifested by the ovarian surface epithelium when it undergoes neoplasia, such as tubal-type epithelium in serous neoplasms, endometrial epithelium in endometrioid tumors, and endocervical epithelium in at least some mucinous neoplasms ${ }^{(6)}$.

PAX8 is a nuclear transcription factor with limited expression in normal and neoplastic tissues in a cell lineage-dependent manner (7). It is a member of the PAX gene family, consisting of nine well-described transcription factors (PAX1-9) (8). PAX8 is important in embryogenesis of the thyroid, Müllerian, and renal/upper urinary tracts, it's also regulates Wilms tumor suppressor gene (WT1) expression ${ }^{(9,10)}$. In the pancreas, PAX proteins play a critical role in islet cell differentiation (11). PAX8, initially detected in ovarian tumors and is characteristic for the epithelial histotypes (serous, clear cell, and endometrioid). So, positive expression of PAX8 represents a strong argument for the confirmation of the origin of ovarian carcinoma arising from the fimbrial area of Fallopian tubes or from an endometriosis foci. Moreover, PAX8 allows the differentiation between Müllerian and nonMüllerian origin which could be derived from a primary tumor in pancreas, colon or mammary glands ${ }^{(12)}$. While the PAX8 expression is useful in the diagnosis of ovarian cancers, it failed to show any prognostic role in an analysis of 148 serous ovarian carcinoma ${ }^{(13)}$.

The objectives of this study were to assess the immunohistochemichal expressions of transcription factor (PAX8) in ovarian surface epithelial tumors (including benign, borderline and malignant) and to study its relationship with the age of the patient, presence or absence of ascites, tumor histopathological type, tumor grade and tumor stage.

\section{Methods}

A retrospective study included a total of 100 ovarian tissue paraffin blocks. Seventy tissue paraffin blocks were assigned as the case group included ovarian tumors obtained from patient who underwent total abdominal hysterectomy and bilateral salpingo-oophorectomy for malignant tumors, and ovarian cystectomy for benign tumors. These blocks were collected from Teaching laboratories of Medical City, AlYarmook Teaching Hospital and private laboratories from January 2015 to April 2017. Thirty tissue paraffin blocks were assigned as the control group included normal ovarian tissue, inclusion cysts, corpus luteal cysts, follicular cysts, endometriosis (chocolate cyst), and fallopian tubes tissue, these blocks were collected from Medical City, Teaching laboratories from November 2016 to January 2017.

The clinico-pathological parameters including (age of patient, ascites, tumor type, and tumor grade and tumor stage) were obtained from patients' admission case sheets and pathology reports.

From each paraffin block, 2 sections were taken, each of $5 \mu \mathrm{m}$ thickness. One was stained with the routine Hematoxylin \& Eosin (H\&E) stain examined and the histopathological diagnosis, tumor histological type and grade according to FIGO system were revised by a pathologist (14). The other section was deparaffinized and rehydrated at room temperature, Antigen retrieval by Antigen retrieval citrate buffers $\mathrm{pH} 6.0$ [DAKO, Denmark] was carried out by microwave $1 \times 10$ 
min then allowed to cool for 20 mins. Mouse monoclonal PAX8 antibody [PAX8R1] (ab53490) (Abcam, UK (dilution $5 \mu \mathrm{g} / \mathrm{ml}$ ) applied to sections and were incubated for an overnight. Expose mouse and rabbit specific HRP/DAB detection IHC kit (ab80436) (Abcam, UK) reagents was used ${ }^{(15)}$. Counterstaining of the sections by Mayer's Hematoxylin stain for 2030 seconds then followed by mounting of the sections by using Roti $^{\circledR}$-Mount Aqua (ROTH, Germany) followed by glass coverslip. Technical negative control was done by the omission of PAX8 antibody.

\section{Interpretation of immunohistochemistry staining and quality control for PAX8}

Immunohistochemically stained slides were reviewed and positive staining was based on the presence of nuclear expression, the staining expressions were scored with assessment of both staining percentage and staining intensity under the light microscopy as follow:

A. The intensity: the intensity of positivity was scored as follows:

$0:$ no staining,

1: weak,

2: moderate,

3: strong.

B. The percentage: The extent of positivity was scored according to the percentage of cells showing positive staining as follows:

- $0 \%$ is 0 , negative

- $1-25 \%$ is $1+$

- $26-50 \%$ is $2+$

- $51-75 \%$ is $3+$

- $76-100 \%$ positive cells is $4+$.

These values [intensity and the percentage] then were multiplied, a final score with possible range of values from 0 to 12 was obtained. The scores $=$ or $>1$ were considered as positive ${ }^{(16)}$.

\section{Statistical analysis}

Numerical data were presented as mean \pm standard error, unpaired t-test was used to compare means between two groups while analysis of variance (ANOVA) was used in case if number of groups was more than two. Categorical data were presented as number and percentage. the comparison of number between different groups was done using Fisher exact test and chi square test. Pearson correlation was done between different parameters and presented as $r$ (correlation coefficient) and $p$ value (level of significance). $P$ value $<0.05$ was considered significant. The software used was Microsoft excel 2016 and SPSS (statistical package for social sciences) version 23.

\section{Results}

Among the 70 cases of ovarian surface epithelial tumors; 13 cases (65\%) of benign tumors showed positive expression for PAX8, borderline tumors, 25 cases $(83.3 \%)$ showed positive expression for PAX8, and the malignant tumors, 17 cases (85\%) showed positive expression for PAX8 marker, while out of 30 cases of control group, only 2 cases (6.7\%) showed positive expression of PAX8. According to that, there is a highly significant difference in the expression of PAX8 between the control group and the case group with the highest expression seen in malignant ovarian surface epithelial tumors $(P<0.001)$ (Table 1$)$. According to semiquantitive scoring system, the control group showed the lowest immunohistchemical expression of PAX8 (Mean=0.1, SE= 0.07), while the ovarian surface epithelial tumors scoring showed higher results, in which malignant ovarian surface epithelial tumors showed the highest immunohistchemical expression of PAX8 (Mean=9.3, SE= 1.01), the borderline tumors immunohistchemical expression of PAX8 was (Mean=6.13, SE= 0.66) and the benign tumors showed the lowest immunohistchemical expression of PAX8 among the ovarian surface epithelial tumors (Mean= 4.5, SE= 0.94), according to that, there is a highly significant difference in the immunohistochemical expression of PAX8 according to the semiquantitive scoring between the control 
Iraqi JMS 2018; Vol. 16(3)

group and the case group with the highest surface epithelial tumors $(P<0.001)$ (Table 2$)$. expression seen in the malignant ovarian

Table 1. Frequency of the positive and negative expression of PAX8 in case and control groups

\begin{tabular}{ccccccc}
\hline \multirow{2}{*}{ Marker } & Expression & $\begin{array}{c}\text { Control } \\
\text { No. (\%) }\end{array}$ & $\begin{array}{c}\text { Benign } \\
\text { No. (\%) }\end{array}$ & $\begin{array}{c}\text { Borderline } \\
\text { No. (\%) }\end{array}$ & $\begin{array}{c}\text { Malignant } \\
\text { No. (\%) }\end{array}$ & P value \\
\hline \multirow{2}{*}{ PAX8 } & Positive & $2(6.7)$ & $13(65.0)$ & $25(83.3)$ & $17(85.0)$ & \multirow{2}{*}{ 0.001** } \\
& Negative & $28(93.3)$ & $7(35.0)$ & $5(16.7)$ & $3(15.0)$ & \\
\hline
\end{tabular}

**: high statistically significant

Table 2. Scores of immunohistochemical expression of PAX8 in control and ovarian tumors

\begin{tabular}{|c|c|c|c|c|c|}
\hline Marker & $\begin{array}{c}\text { Control } \\
\mathrm{N}=30 \\
\text { Mean } \pm \mathrm{SE}\end{array}$ & $\begin{array}{c}\text { Benign } \\
\mathrm{N}=20 \\
\text { Mean } \pm S E\end{array}$ & $\begin{array}{c}\text { Borderline } \\
\mathrm{N}=\mathbf{3 0} \\
\text { Mean } \pm \mathrm{SE}\end{array}$ & $\begin{array}{c}\text { Malignant } \\
\mathrm{N}=\mathbf{2 0} \\
\text { Mean } \pm \mathrm{SE}\end{array}$ & $P$ value \\
\hline PAX8 & $0.1 \pm 0.07$ & $4.5 \pm 0.94$ & $6.13 \pm 0.66$ & $9.3 \pm 1.01$ & $<0.001 * *$ \\
\hline
\end{tabular}

According to the semiquantitive scoring PAX8 showed a high significant difference among benign, borderline and malignant ovarian surface epithelial tumors in relation to histopathological types $(P<0.001)$ except for the mucinous tumors which showed nonsignificant difference in the immunohistochemical expression of PAX8 according to the semiquantitive scoring $(P=0.641)$. Also, a highly statistically significant difference in the immunohistochemical expression of PAX8 according to the semiquantitive scoring among serous, mucinous and others histological subtypes in relation to histological classification of ovarian surface epithelial tumors in to benign, borderline and malignant $(P<0.001)$ (Table 3$)$.

Among the 70 cases of ovarian epithelial tumors collected during the study, papillary serous cystadeno-carcinoma and serous adenocarcinoma showed the highest immunohistochemical expression score of PAX8 (Mean= 11.6, SE= 0.4) (Figure 1), followed by other histopathological subtypes [endometrioid adenocarcinoma (Figure 2), malignant brenner tumors, clear cell carcinoma, and transitional cell carcinoma] (Mean=11.0, SE= 0.63), while in the mucinous cystadenocarcinoma was with (Mean $=1.0, \mathrm{SE}=$ 1.0) (Table 3).

PAX8 expression in borderline tumors was lower in serous tumors (Mean $=8.16, \mathrm{SE}=0.45$ ) (Figure 3), mucinous (Mean=0.43, SE=0.3) and other histological subtypes (Mean $=6.5, \mathrm{SE}=$ 0.5) (Figure 4). Benign serous tumors PAX8 expression was (Mean $=8.2, \mathrm{SE}=0.66$ ) while benign mucinous cystadenoma and the other histological category showed the lowest expression of PAX8 (Table 3).

Regarding other clinicopathological parameters, PAX8 showed a highly significant relation with the increment of tumor grade $(r=$ 0.769 and $P<0.001)$. PAX8 showed a nonsignificant relation with increase of the age $(r=$ $0.147, P=0.225)$, with tumor stage $(r=0.543, P$ $=0.013$ ) (Table 4).

There was no significant association found with the presence of ascites $(P=0.446)$ (Table 5). 
Table 3. Score of the expression of PAX8 in relation to histopathological type of ovarian surface epithelial tumors

\begin{tabular}{ccccc}
\hline Histopathology & $\begin{array}{c}\text { Benign } \\
\text { Mean } \pm \text { SE }\end{array}$ & $\begin{array}{c}\text { Borderline } \\
\text { Mean } \pm \text { SE }\end{array}$ & $\begin{array}{c}\text { Malignant } \\
\text { Mean } \pm \text { SE }\end{array}$ & P value \\
\hline Serous & $8.2 \pm 0.66$ & $8.16 \pm 0.45$ & $11.6 \pm 0.4$ & $<0.001^{* *}$ \\
Mucinous & $0.25 \pm 0.25$ & $0.43 \pm 0.3$ & $1.0 \pm 1.0$ & $0.641^{\mathrm{NS}}$ \\
Others & $1.17 \pm 0.75$ & $6.5 \pm 0.5$ & $11.0 \pm 0.63$ & $<\mathbf{0 . 0 0 1 * *}$ \\
\hline P value & $<\mathbf{0 . 0 0 1 * *}$ & $<\mathbf{0 . 0 0 1 * *}$ & $<\mathbf{0 . 0 0 1 * *}$ & \\
\hline
\end{tabular}

$* *$ : high statistically significant, NS: Non-statistically significant. Others = Endometrioid tumors, Brenner tumors, clear cell tumors, and transitional carcinoma

Table 4. Relation of PAX8 expression with age, tumor grading, tumor staging

\begin{tabular}{ccc}
\hline Parameter & \multicolumn{2}{c}{ PAX8 score } \\
\hline Age (years) & $\mathbf{r}$ & $\mathbf{P}$ \\
\hline Tumor grade (FIGO) & 0.147 & $0.225^{\mathrm{NS}}$ \\
Tumor stage (FIGO) & 0.769 & $<\mathbf{0 . 0 0 1 ^ { * * }}$ \\
\hline
\end{tabular}

**: high statistically significant, NS: Non-statistically significant.

Table 5. Relation of PAX8 expression scores with presence of ascites

\begin{tabular}{cccc}
\hline & Ascites & & \\
Marker & Positive & Negative & P value \\
& $\mathrm{N}=33$ & $\mathbf{N}=37$ & \\
\hline PAX8 score & $7.0 \pm 0.82$ & Mean \pm SE & $0.446^{\mathrm{NS}}$ \\
\hline
\end{tabular}

NS: Non-statistically significant

\section{Discussion}

PAX8 plays an important role in the tumorigenic phenotype of ovarian cancer cells (8). Among the 70 cases of ovarian epithelial tumors; 13 cases (65\%) of benign tumors showed positive expression for PAX8, borderline tumors, 25 cases (83.3\%) showed positive expression for PAX8, and the malignant tumors, 17 cases (85\%) showed positive expression for PAX8 marker. For the control group; out of 30 cases, only 2 cases (6.7\%) showed positive expression of PAX8. According to the results of this study, there is a highly significant difference in the expression of PAX8 between the control group and the case group with the highest expression seen in malignant ovarian surface epithelial tumors ( $P$ $<0.001$ ).

The current study showed much lower level expression of PAX8 in control group than a study performed by Adler et al. which showed that PAX8 was expressed in $44 \%$ of the ovarian surface epithelial cells, while it was negative in $56 \%$ of normal ovarian tissues and all stromal tissues (17). 


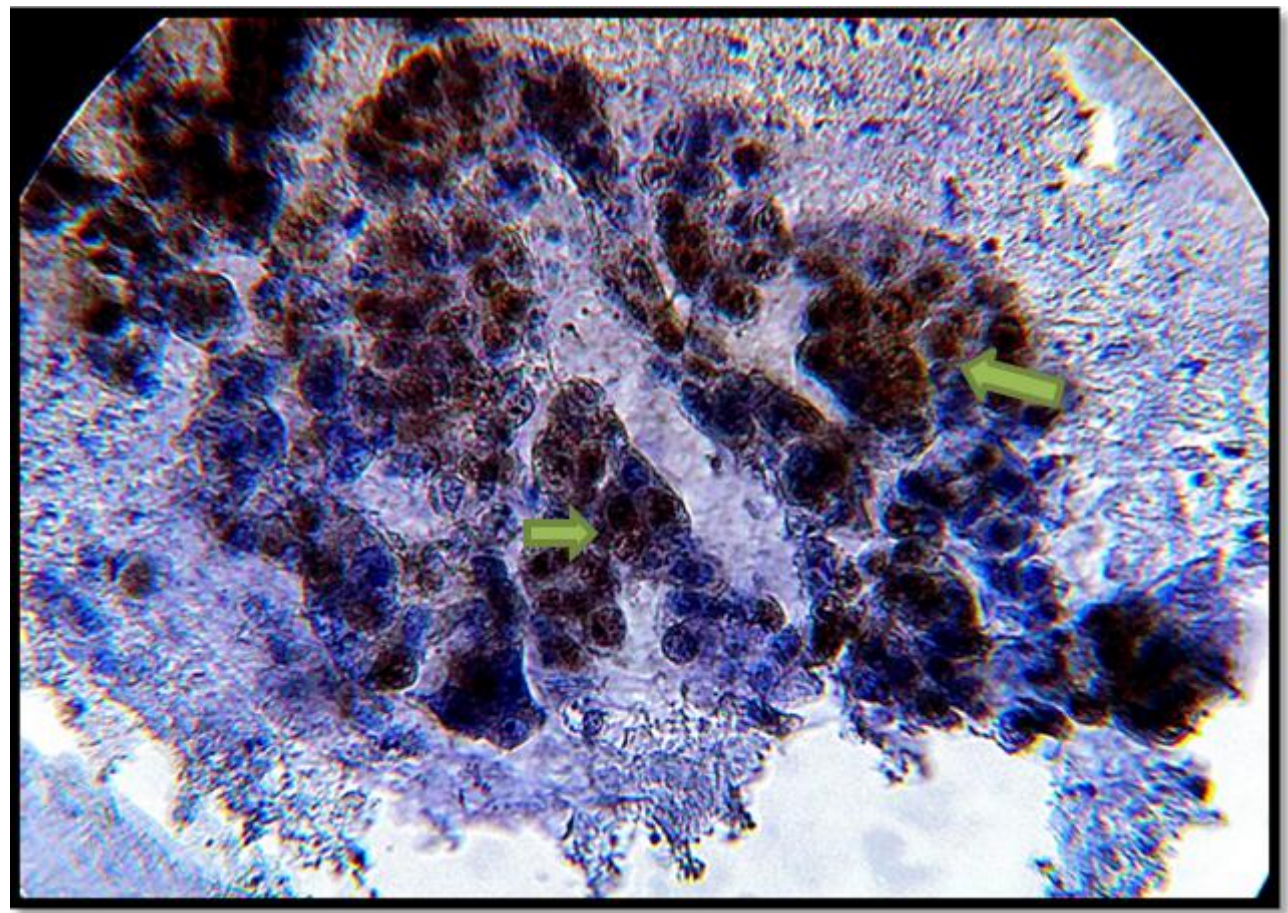

Figure 1. Serous adenocarcinoma (FIGO grade III) tissue section showing positive brown nuclear expression of PAX8 monoclonal antibody of $95 \%$ of epithelial cells with strong intensity and score of 12 (arrows) (40x)



Figure 2. Endometrioid adenocarcinoma (FIGO grade I) tissue section showing positive brown nuclear expression of PAX8 monoclonal antibody of $85 \%$ of epithelial cells with moderate intensity and score of 8 (arrows) (40x) 


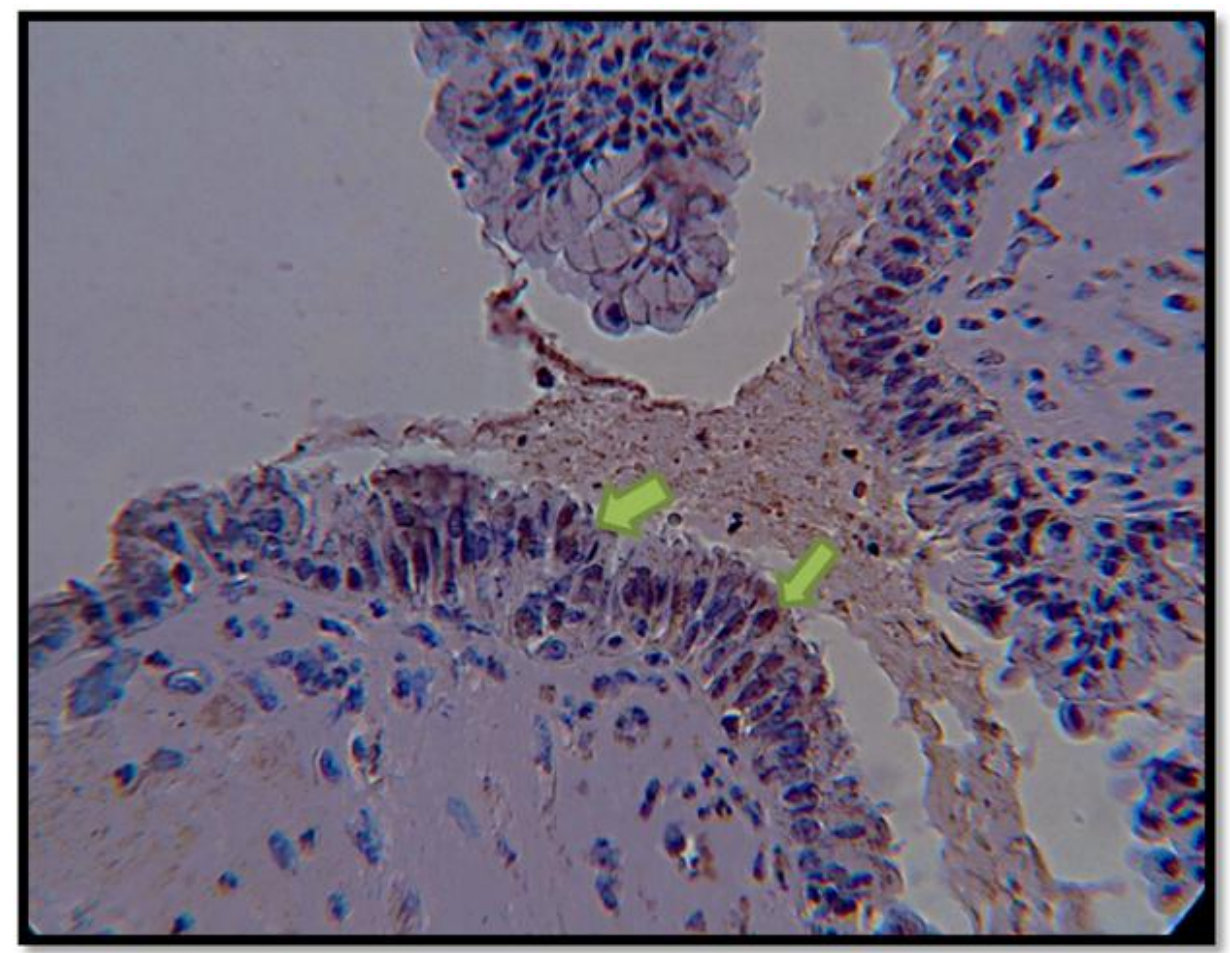

Figure 3. Borderline serous tumor tissue section showing positive brown nuclear expression of PAX8 monoclonal antibody of $45 \%$ of epithelial cells with moderate intensity and score of 4 (arrows) (40x)

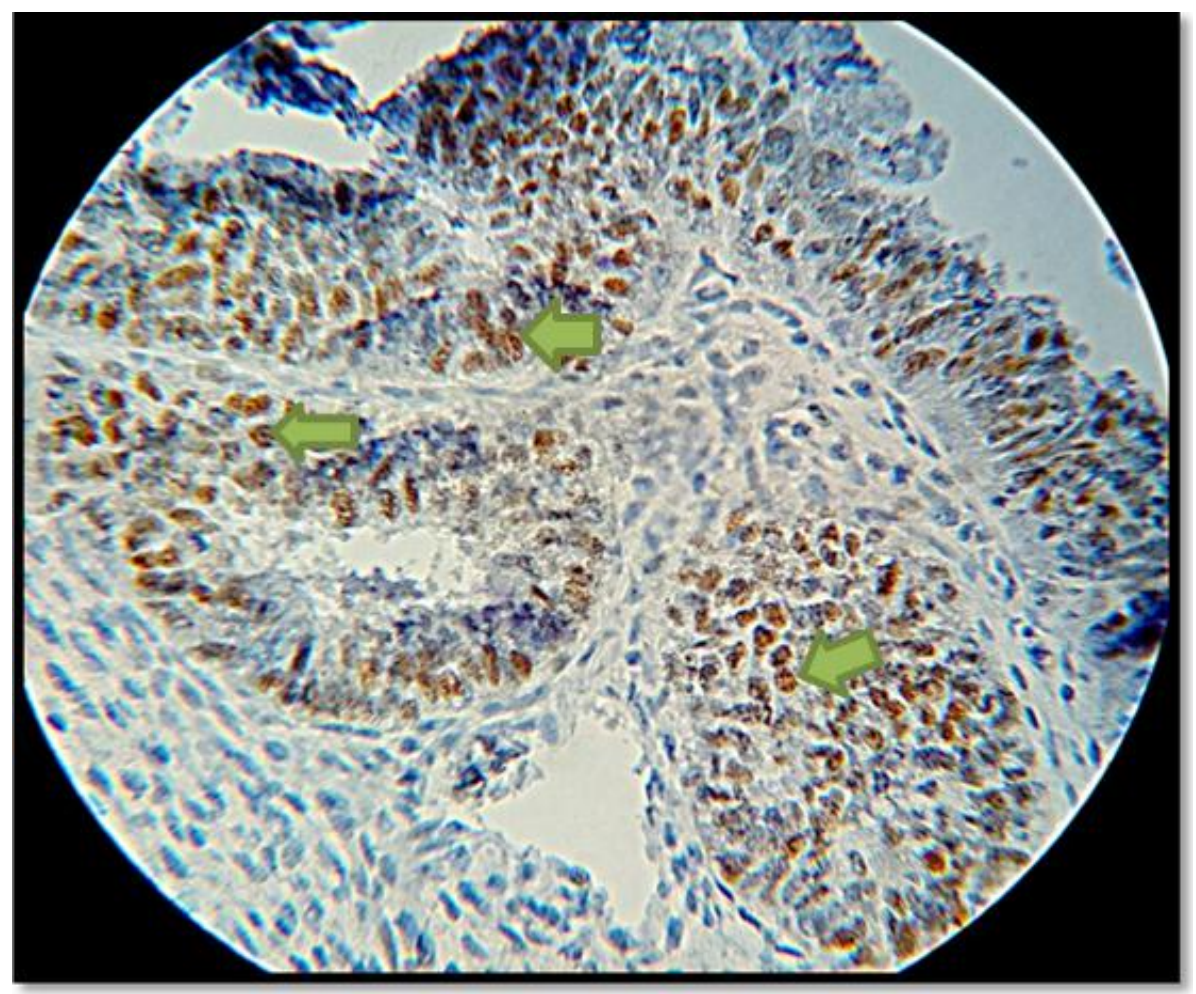

Figure 4. Borderline endometrioid tissue section showing positive brown nuclear expression of PAX8 monoclonal antibody of 55\% of epithelial cells with moderate intensity and score of 6 (arrows) (40x) 
This may be attributed to that in Adler et al. ${ }^{(17)}$ study, PAX8 expression was evaluated in 27 histologically normal ovaries that contained ovarian surface epithelial cells present on the surface while in current study not all the 30 sections contained epithelial surface cells, so the variation in the level may be attributed to types of cases included in the study, geographical factor, difference in clone of marker used, and immonohistochemical technique. Parallel to our study results, normal ovarian surface epithelium was uniformly negative in the Bowen et al. ${ }^{(18)}$ study analysis, which also demonstrate that PAX8 is expressed in the epithelial ovarian cancer cell line (OVCAR-3) and this support the hypothesis that PAX8 plays equivalent role in the development of epithelial ovarian cancer. In contrast to our study normal ovarian surface epithelium was positive in $62 \%$ of Turkish study by Ozcan et al. (20), which showed that PAX8 are constantly expressed in normal or non-neoplastic tissue of mullerian origin and parallel to this study results, Ozcan et al. (19) demonstrated that strong and diffuse staining of PAX8 was found in most cases of the primary ovarian epithelial tumors except for the mucinous type. The differences attributed to differences in sample size in which Ozcan et al study involved very large number samples, multiple organs were involved, difference in clone of marker used, and immonohistochemical technique.

In the current study among the 70 cases collected, papillary serous cystadenocarcinoma and serous adenocarcinoma showed positive expression of PAX8 with the highest immunohistochemical expression level for PAX8. Mucinous tumors showed the lowest immunohistochemical expression levels of PAX8 among the ovarian surface epithelial tumors. A highly significant association of PAX8 expression in relation to histopathological types $(P<0.001)$ except for the mucinous tumors which showed statistically nonsignificance $(P=0.641)$.

An American study performed by Nonaka et al. found that PAX8 serves as an important marker for discriminating ovarian nonmucinous surface epithelial carcinomas, in which PAX8 reaction was found in $87.1 \%$ ovarian surface epithelial carcinomas, the commonest was serous papillary carcinomas, followed by endometrioid carcinomas, clear cell carcinomas, and mucinous carcinomas ${ }^{(10)}$. The results of the immunohistochemistry stains of this study was $85 \%$, which is parallel to Nonaka et al. (10) study, in which $87.1 \%$ of ovarian carcinomas positive for PAX8. Most of the tumors in the current study were of serous type, followed by ovarian non-mucinous tumors subtypes, which was parallel to Nonaka et al. (10) study. Serous malignant tumors showed highest expression levels of PAX8 followed by endometrioid carcinoma and clear cell carcinoma. The current study was parallel to Nonaka et al. (10) study, in which the mucinous carcinoma type showed the lower expression levels of PAX8. In Ozcan et al. (19) study PAX8 showed strong and diffuse staining in most cases of all histologic subtypes, except in mucinous tumors, this study agrees with the current one ${ }^{(19)}$.

The current study showed a non-significant relation between the expression of PAX8 and increase of the age $(r=0.147$ and $P=0.225)$. Due to the limited number of articles that consider the relation of PAX8 and age parameters we reviewed its relation in other organs, in which we found that the results of the current study go parallel to the Austrian study performed by Brunner et al. on the immunoexpression of PAX8 in endometrial cancer and its relation to high grade carcinoma, which found that no significant correlation was observed between PAX8 and patient age (21). Also parallel to other American study performed by Long et al. on PAX8 expression in well-differentiated pancreatic endocrine tumors, and its correlation with clinicopathologic features showed that PAX8 expression was not correlated with patient age (11).

Regarding the relation of PAX8 expression semiquantitive scoring and tumor grade in the current study, there is high significant relation between the expression of PAX8 and increase of tumor grade $(r=0.769$ and $P<0.001)$. Ozcan et al. mentioned that PAX8 was much more pronounced for high-grade carcinomas (99\%) than for cystadenomas/borderline tumors 
(96\%), however they noted that the PAX8 expression is independent of tumor grade, at least for serous tumors (19). Laury et al. mentioned that in the ovary about $92 \%$ of endometrioid carcinomas were positive for PAX8, and the higher the tumor grade, the more diffuse and stronger PAX8, however, they mentioned that there was no obvious relation with tumor grade in mullerian carcinomas as the majority of tumors of various subtypes were positive for PAX8 regardless tumor grade (9). While a positive correlation was observed between PAX8 and histologic grade in both Brunner et al. (20) $(P=0.02)$ and MhawechFauceglia et al. (21) $(\mathrm{P}=0.002)$ in endometrial carcinoma.

Regarding the relation of PAX8 immunohistochemical expression and tumor stage, there was no significant relation between the PAX8 expression and increase of tumor stage $(r=0.433$ and $P=0.057)$. The results of this study go parallel with the results of an American study performed by MhawechFauceglia et al., which found that though PAX8 was expressed in $61 \%$ cases of 148 serous with late stage ovarian carcinoma, there was no association between PAX8 and tumor stage (22). Also, was parallel to the Malaysian study performed by Rhodes et al. ${ }^{(23)}$, which noticed that the expression of PAX8 in the ovarian serous and endometrioid subtypes was not associated with stage and they regard this to the small number of cases, and the majority being with late stage.

Regarding the relation of PAX8 expression and presence of ascites, among the 70 cases of ovarian surface epithelial tumors, there were 33 cases present with ascites and PAX8 expression score of (Mean $=7.0, \mathrm{SE}=0.82$ ). According to that, there is non- significant relation between the expression of PAX8 and presence of ascites with $P=0.446$. One study performed by Ayantunde and Parsons mentioned that ascites is an independent prognostic factor at presentation, and $>1 / 3$ of women with ovarian cancer will develop ascites during their disease course, and this is not limited to a specific histological subtype (24). Also, the American study performed by Mhawech-Fauceglia et al. found that there was no correlation between PAX8 expression level and tumor stage, and it failed to predict a value in disease outcome ${ }^{(21)}$. This support that there is no correlation between PAX8 expression levels and the presence of ascites.

In conclusion, the current study shows a highly significant differences in the immunohistochemical expression of PAX8 in ovarian surface epithelial tumors tissues compared to control groups, which in turn reflects the important role of PAX8 in ovarian carcinoma development. Also, PAX8 immunohistochemical expression was highest in ovarian epithelial tumors of serous type and lowest in the mucinous type, so that it can be used as an important marker for discriminating ovarian non-mucinous from mucinous tumors. The significant relation of PAX8 expression with higher tumor grade, may refer to the possible role of PAX8 in the development and differentiation of ovarian malignant surface epithelial tumor, which in turn suggests that PAX8 may promote the progression of ovarian cancer, through activation of the anti-apoptotic genes which promote increased cell proliferation through upregulation of epithelial-mesenchymal transition (EMT).

\section{Acknowledgments}

Authors are thankful to everyone associated with this work starting from the staff of Laboratory of Pathology Department in the Medical College of Al-Nahrain University and ending with all doctors \& staff of Teaching Laboratories of Medical City, Al-Yarmook Teaching Hospital for their valuable support and advice.

\section{Authors' contribution}

Dr. Al-Shami collected the cases data, performed the routine H\&E and the immunohistochemical technique and analyzed the results of the study. Dr. Qasim helped in study design and supervising the work. Dr. Hassan participated in the immunohistochemical scoring of the histopathological sections.

\section{Conflict of interest}

The authors declare no conflict of interest. 


\section{Funding}

The research working funding was provided by the authors.

\section{References}

1. Permuth-Wey J, Sellers T. Epidemiology of ovarian cancer. In: Verma M (ed). Cancer epidemiology: volume 2 modifiable factors. Switzerland: Springer Nature, Humana Press; 2009. p. 413-37. doi: 10.1007/978-1-60327-492-0_20.

2. Konecny G, Wang C, Hamidi H, et al. Prognostic and therapeutic relevance of molecular subtypes in highgrade serous ovarian cancer. J Natl Cancer Inst. 2014; 106(10). pii: dju249. doi: 10.1093/jnci/dju249.

3. Acharya U, Mookiah M, Vinitha Sree S, et al. Evolutionary algorithm-based classifier parameter tuning for automatic ovarian cancer tissue characterization and classification. Ultraschall Med. 2014; 35(3): 237-45. doi: 10.1055/s-0032-1330336.

4. Iraqi cancer registry 2011. Iraqi Ministery of Health. 2011. p. 1-10.

5. Abdul Ghany $M$, Khattab $Y$, Al-Kurtas $M$. Immunohistochemical expression of CD44v6 and P53 status in borderline and malignant ovarian surface epithelial tumors. A clinico-pathologic study. Iraqi JMS. 2016; 14: 7-14.

6. Philip C, Young R. Female reproductive system and peritoneum - ovarian surface epithelial-stromal tumors. In: Mills E, Carter D, Greenson J, et al. (eds) Sternberg's Diagnostic Surgical Pathology. $5^{\text {th }}$ ed. Lippincott Williams \& Wilkins; 2012. p. 2278-309.

7. Tong GX, Devaraj K, Hamele-Bena D, et al. PAX8: a marker for carcinoma of müllerian origin in serous effusions. Diagn Cytopathol. 2011; 39(8): 567-74. doi: $10.1002 / d c .21426$.

8. Di Palma T, Lucci V, de Cristofaro T, et al. A role for PAX8 in the tumorigenic phenotype of ovarian cancer cells. BMC Cancer. 2014; 14: 292. doi: 10.1186/14712407-14-292.

9. Laury A, Perets $\mathrm{R}$, Piao $\mathrm{H}$, et al. A comprehensive analysis of PAX8 expression in human epithelial tumors. Am J Surg Pathol. 2011; 35(6): 816-26. doi: 10.1097/PAS.0b013e318216c112.

10. Nonaka D, Chiriboga L, Soslow R. Expression of PAX8 as a useful marker in distinguishing ovarian carcinomas from mammary carcinomas. Am J Surg Pathol. 2008; 32(10): 1566-71. doi: 10.1097/PAS.0b013e318216c112.

11. Long $K$, Srivastava $A$, Hirsc $M$, et al. PAX8 expression in well-differentiated pancreatic endocrine tumors: correlation with clinicopathologic features and comparison with gastrointestinal and pulmonary carcinoid tumors. Am J Surg Pathol. 2010; 34(5): 7239. doi: 10.1097/PAS.0b013e3181da0a20.

12. Liliac $L$, Carcangiu M, Canevari $S$, et al. The value of PAX8 and WT1 molecules in ovarian cancer diagnosis. Rom J Morphol Embryol. 2013; 54(1): 17-27.
13. Davidson B, Tropé CG. Ovarian cancer: diagnostic, biological and prognostic aspects Womens Health (Lond). 2014; 10(5): 519-33. doi: 10.2217/whe.14.37.

14. Colombo N, Peiretti M, Parma G, et al. Newly diagnosed and relapsed epithelial ovarian carcinoma: ESMO clinical practice guidelines for diagnosis, treatment and follow-up. Ann Oncol. 2010; 21 Suppl 5: v23-30. doi: 10.1093/annonc/mdq244.

15. Abcam. Expose mouse and rabbit specific HRP/DAB detection IHC kit (ab80436). 2016:1-3.

16. Yemelyanova A, Gown A, Wu LF, et al. PAX8 expression in uterine adenocarcinomas and mesonephric proliferations. Int J Gynecol Pathol. 2014; 33(5): 492-9. doi: 10.1097/PGP.0b013e3182a54afa.

17. Adler E, Mhawech-Fauceglia P, Gayther S, et al. PAX8 expression in ovarian surface epithelial cells. Hum Pathol. 2015; 46(7): 948-56. doi: 10.1016/j.humpath.2015.03.017.

18. Bowen N, Logani S, Dickerson E, et al. Emerging roles for PAX8 in ovarian cancer and endosalpingeal development. Gynecol Oncol. 2007; 104(2): 331-7. doi: 10.1016/j.ygyno.2006.08.052.

19. Ozcan A, Liles N, Coffey D, et al. PAX2 and PAX8 expression in primary and metastatic müllerian epithelial tumors: a comprehensive comparison. Am J Surg Pathol. 2011; 35(12): 1837-47. doi: 10.1097/PAS.0b013e31822d787c.

20. Brunner A, Riss $P$, Heinze $G$, et al. Immunoexpression of PAX 8 in endometrial cancer: relation to highgrade carcinoma and p53. Int J Gynecol Pathol. 2011; 30(6): 569-75. doi: 10.1097/PGP.0b013e31821ac6c3.

21. Mhawech-Fauceglia $P$, Wang $D$, Samrao $D$, et al. PairBox (PAX8) protein-positive expression is associated with poor disease outcome in women with endometrial cancer. Br J Cancer. 2012; 107(2):370-4. doi: 10.1038/bjc.2012.241.

22. Mhawech-Fauceglia $P$, Wang $D$, Samrao $D$, et al. Pair Box 8 (PAX8) protein expression in high grade, late stage (stages III and IV) ovarian serous carcinoma. Gynecol Oncol. 2012; 127(1): 198-201. doi: 10.1016/j.ygyno.2012.06.012.

23. Rhodes A, Vallikkannu $N$, Jayalakshmi P. Expression of WT1 and PAX8 in the epithelial tumours of Malaysian women with ovarian cancer. Br J Biomed Sci. 2017; 74(2): 65-70. doi: 10.1080/09674845.2016.1220709.

24. Ayantunde A, Parsons S. Pattern and prognostic factors in patients with malignant ascites: a retrospective study. Ann Oncol. 2007; 18(5): 945-9. DOI: 10.1093/annonc/mdl499.

\footnotetext{
Correspondence to Dr. Samar A. Al-Shami

E-mail: dr.samaralshami1@gmail.com dr.samaralshami1@colmed-alnahrain.edu.iq Received Nov. 14 2017 Accepted Feb. $8^{\text {th }} 2018$
} 